\title{
Stochastic analysis of a single-unit system with repairman having multiple vacations
}

\author{
Ram Niwas $^{\# 1}$ and M.S. Kadyan ${ }^{\# 2}$ \\ ${ }^{\# 1}$ Department of Statistics, GGDSD College, Panjab University, Chandigarh-160014, India \\ ${ }^{\# 2}$ Department of Statistics \& O.R., Kurukshetra University, Kurukshetra-136119, India
}

\begin{abstract}
:
This paper considers a repairable system with a repairman, who can take multiple vacations. If the system fails and the repairman is on vacation, it will wait for repair until the repairman is available. When the repairman comes back from his vacation, he starts repair immediately if the system wait for repair otherwise he will wait idly for fail arrival. Using Supplementary Variable Technique, the various expressions which depict the behavior of the system such as reliability of the system, Mean Time to System Failure (MTSF), availability, busy, vacation, idle times of the repairman and profit function have been derived. Finally, to effectiveness the proposed approach, the effect of the parameters of the system has been analyzed on to the system reliability and expected profit through an illustrative example.
\end{abstract}

Keywords: Stochastic analysis, single-unit system, multiple vacations.

Corresponding Author: Ram Niwas

\section{Introduction:}

Reliability and availability is good assessor of a repairable system's performance and play an important role for manufacturing and industrial systems. In traditional repairable systems, it is assumed that the repairman remains idle until a failure occurs. However, the repairman can affect the economic benefit of the system directly or indirectly. Therefore, to improving the profit of the system, the repairman might take a sequence of vacations in the idle time. Repairman's vacations are useful for the system in which the repairman wants to utilize his idle time for different purposes discussed by Levy and Yechiali [16]. When there is no failed unit in the system, the repairman leaves for a vacation or may take other assigned job, which can have great influence on performance of the system.

Vacation model originally arises in queueing theory; Ke [7] introduced the concept of vacation policy into modeling analysis of queue system in which two standard vacation policies were defined: single and multiple vacations. Also, works on vacation models have been reported by Doshi [2], Lee [6], Takagi [5], Tian and Zhang [13], and Ke et al. [8] provided a summary of most recent works on vacation queueing system in the past decades. In reliability theory, Vacation concept was linked by Su and Shi [1] and discussed $n$-unit series system with multiple vacations. Inspired by this reliability vacation model, various researchers introduced vacation models into repairable systems. Wu et al. [15] analyzed reliability of a K-out-of-n: $\mathrm{G}$ repairable system with multiple vacations. A warm maintenance repairable system with continuous switch and multiple vacations for repairman discussed by Zhang and $\mathrm{Wu}$ [12]. Qiao and Guo [9] developed a repairable system with repairman vacation and warning device which can send an alarm when the system is not in good condition. A deteriorating system with single vacation was analyzed by Sun and Ma [4]. Yuan and $\mathrm{Xu}$ [10] evaluated an optimal replacement policy of a deteriorating system with its repairman having multiple vacations with the assumption that system cannot be repaired as good as new after failure. 
But to improve the performance of the system, it is more beneficial to use a system in which repairs are perfect i.e., the unit works as good as new after repair. Keeping these views in mind here we proposed a single-unit repairable system with repairman having multiple vacations by using supplementary variable technique [3, $11 \& 14]$. In the proposed approach, the unit works as good as new after its repair. The remainder of this paper is described in different sections as: Section 2 gives the description of the system containing the assumptions of the model, state-specifications, and notations related to the proposed system model, Section 3 represents the system analysis in which different system performance measures are computed such as reliability of the system and MTSF, Section 4 shows the results and discussion with special case containing availability of the system, busy time, vacation time, idle time of the repairman and profit analysis of the system and provides a numerical result for these special cases. Finally, Section 5 represents concluding remarks.

\section{Description of the System}

\subsection{Assumptions:}

1) The system consists of a single machine and single repairman, the machine is new and starts to work at the initial time $t=0$ and the repairman takes his first vacation after the system has started.

2) If the machine fails during the repairman's vacation, the system is in the state of waiting for repair.

3) The repairman goes for vacation after repairing the failed machine.

4) The machine works as good as new after its repair.

5) The distribution of failure time of a machine and arrival time of a repairman after his vacations is taken as negative exponential while the repair time is considered as arbitrary.

\subsection{State-Specifications:}

Let $\{N(t), t \geq 0\}$ be a stochastic process with state space $E=\{0,1,2,3\}$. The states are defined as follows:

0: $\quad$ The system is working at time $t$, the repairman is on vacation;

1: $\quad$ The system is working and the repairman wait idly in the system:

2: $\quad$ The system is failed at time $t$, the repairman is on vacation:

3: $\quad$ The system is failed at time $t$, the repairman is repairing the failed machine.

\subsection{Notations:}

$\lambda: \quad$ Constant failure rate of the machine.

$a: \quad$ Constant arrival rate of the repairman after his vacation.

$\mu(x), S(x)$ : $\quad$ Repair rate of the failed machine and probability density function, for elapsed repair time $\mathrm{x}$.

$p_{0}(t)$ : $\quad$ Probability density that at time $t$, the system is in good/working state and the repairman is on his vacation.

$p_{1}(t)$ : $\quad$ Probability density that at time $\mathrm{t}$, the system is in good/working state and the repairman wait idly in the system.

$p_{2}(t)$ : $\quad$ Probability density that at time $\mathrm{t}$, the system is in failed state and the repairman is on his vacation.

$p_{3}(x, t)$ : Probability density that at time $\mathrm{t}$, the system is in failed state and machine is under repair with elapsed repair time $\mathrm{x}$. 
$p(s): \quad$ Laplace transform of function $p(t)$.

$S(x): \quad \mu(x) \exp \left[-\int_{0}^{x} \mu(x) d x\right]$

\section{System Analysis:}

The system consists of a single-unit in which there is a single repairman, who can take multiple vacations. Consider that at initially, the system is in good working condition and repairman is on vacation. If the machine fails during the repairman is on vacation, the system is in the state of waiting for repair. When the repairman comes back from his vacation, there are two cases: one is the system in the state of waiting for repair, he starts its repair immediately; the other is the machine being running, he will wait idly in the system for occurrence of failure/fail arrival, upon which he starts repairing it immediately. But, when the failed machine has been repaired, the repairman will go for a vacation again. It has been assumed the failure time of a machine and arrival time of a repairman after his vacations are follow a negative exponential distribution while the repair time distribution is considered as arbitrary.

\subsection{Formulation of mathematical model}

Based on the above description, we can formulate the difference-differential equations by using the probabilistic arguments of each state of the system and are summarized as follows:

$$
\begin{aligned}
& {\left[\frac{d}{d t}+a+\lambda\right] p_{0}(t)=\int_{0}^{\infty} \mu(x) p_{3}(x, t) d x} \\
& {\left[\frac{d}{d t}+\lambda\right] p_{1}(t)=a p_{0}(t)} \\
& {\left[\frac{d}{d t}+a\right] p_{2}(t)=\lambda p_{0}(t)} \\
& {\left[\frac{\partial}{\partial t}+\frac{\partial}{\partial x}+\mu(x)\right] p_{3}(x, t)=0}
\end{aligned}
$$

Boundary Condition:

$$
p_{3}(0, t)=a p_{2}(t)+\lambda p_{1}(t)
$$

Initial Conditions:

$$
p_{i}(0)= \begin{cases}1 ; & i=0 \\ 0 & ; i \neq 0\end{cases}
$$

\subsection{Solution of the equations:}

In order to solve the above formulated Eqs. (1) - (5), we use the Laplace transforms corresponding to initial condition given in Eq. (6) and get

$$
\begin{aligned}
& {[s+a+\lambda] p_{0}(s)=1+\int_{0}^{\infty} \mu(x) p_{3}(x, s) d x} \\
& {[s+\lambda] p_{1}(s)=a p_{0}(s)} \\
& {[s+a] p_{2}(s)=\lambda p_{0}(s)} \\
& {\left[\frac{d}{d x}+s+\mu(x)\right] p_{3}(x, s)=0}
\end{aligned}
$$


$p_{3}(0, s)=a p_{2}(s)+\lambda p_{1}(s)$

Thus, by integrating Eq. (10) and further using Eq. (11) we get

$p_{3}(x, s)=\left[a p_{2}(s)+\lambda p_{1}(s)\right] \exp \left(-s x+\int_{0}^{x} \mu(x) d x\right)$

Further, by using Eqs. (8), (9) and (12) Eq. (7) yields

$[s+a+\lambda] p_{0}(s)=1+\left[\frac{a \lambda}{(s+a)}+\frac{a \lambda}{(s+\lambda)}\right] p_{0}(s) S(s)$

$p_{0}(s)=\frac{1}{T(s)}$

Where $T(s)=\frac{[(s+a+\lambda)(s+a)(s+\lambda)-a \lambda(2 s+a+\lambda) S(s)]}{(s+a)(s+\lambda)}$

Using Eq. (13), the Eqs. (8) and (9) yields

$$
\begin{aligned}
& p_{1}(s)=\frac{a}{(s+\lambda) T(s)} \\
& p_{2}(s)=\frac{\lambda}{(s+a) T(s)}
\end{aligned}
$$

Now, the Laplace transform of the probability that the system is in the failed state and under repair is given by

$$
p_{3}(s)=\int_{0}^{\infty} p_{3}(x, s) d x=\left[\frac{a \lambda(2 s+a+\lambda)}{(s+a)(s+\lambda)}\right] \frac{(1-S(s))}{s T(s)}
$$

It is worth noticing that

$$
p_{0}(s)+p_{1}(s)+p_{2}(s)+p_{3}(s)=\frac{1}{s}
$$

\subsection{Evaluation of Laplace transforms of up and down state probabilities}

The Laplace transforms of the probabilities that the system is in up (i.e. good State) and down (i.e. failed State) at time $t$ are as follows

$$
\begin{aligned}
& A_{v}(s) \text { or } P_{u p}(s)=p_{0}(s)+p_{1}(s) \\
& P_{u p}(s)=\frac{1}{T(s)}\left[\frac{(s+a+\lambda)}{(s+\lambda)}\right] \\
& P_{\text {down }}(s)=p_{2}(s)+p_{3}(s) \\
& P_{\text {down }}(s)=\frac{1}{T(s)}\left[\frac{\lambda}{(s+a)}+\frac{\lambda a(2 s+a+\lambda)}{(s+a)(s+\lambda)}\left(\frac{1-S(s)}{s}\right)\right]
\end{aligned}
$$

\subsection{Reliability of the system:}

Reliability $R(t)$ is the probability that the system functions well in a specified period of time. Using the method similar to that in sub section 3.1, the difference- differential equations for reliability are

$$
\left[\frac{d}{d t}+a+\lambda\right] p_{0}(t)=0
$$


$\left[\frac{d}{d t}+\lambda\right] p_{1}(t)=a p_{0}(t)$

Using the initial conditions, the solution can be written as

$$
\begin{aligned}
& p_{0}(s)=\frac{1}{(s+a+\lambda)} \\
& p_{1}(s)=\frac{a}{(s+a+\lambda)(s+\lambda)} \\
& R(s)=p_{0}(s)+p_{1}(s)=\frac{1}{(s+\lambda)}
\end{aligned}
$$

Taking inverse Laplace transform, we get $R(t)=\exp (-\lambda t)$

Now, based on Eq. (25), the Mean Time to System Failure (MTSF) is defined as:

$$
\begin{aligned}
& \text { MTSF }=\int_{0}^{\infty} R(t) d t \\
& =\int_{0}^{\infty} \exp (-\lambda t) d t=\left(\frac{1}{\lambda}\right)
\end{aligned}
$$

\section{Results and discussions:}

In this section, firstly, we have deduced the expression of the availability of the system, busy, vacation, idle times of the repairman and the profit analysis by taking a particular case of the distribution function of the component of the system. Further, we have analyzed the performance of the system with respect to the various affecting parameters of the system which affects the system performance directly or indirectly. For it, the effect of the parameters namely failure rate $(\lambda)$ and arrival rate of the repairman $(a)$ on to the system reliability and the expected profit have been deducted. The results corresponding to these are summarized in Table 1 and Table 2 respectively for the reliability and the expected profit.

\subsection{Availability of the system $A_{v}(t)$ :}

Let $A_{v}(t)$ is the probability that the system is operating satisfactorily at time $t$. Assume that the repair time follow negative exponential distribution i.e., $S(s)=\frac{\mu}{(s+\mu)}$ where $\mu$ is constant repair rate. Putting these values in Eqs. (13)-(17) we get

$$
\begin{aligned}
& p_{0}(s)=\frac{1}{I(s)} \\
& \text { Where } I(s)=\left[\frac{(s+a+\lambda)(s+a)(s+\lambda)(s+\mu)-a \mu \lambda(2 s+a+\lambda)}{(s+a)(s+\lambda)(s+\mu)}\right] \\
& p_{1}(s)=\left[\frac{a}{(s+\lambda) I(s)}\right] \\
& p_{2}(s)=\left[\frac{\lambda}{(s+a) I(s)}\right]
\end{aligned}
$$


DOI: https://dx.doi.org/10.26808/rs.ca.i8v1.14 International Journal of Computer Application (2250-1797)

Issue 8 Volume 1, January- February 2018

$$
\begin{aligned}
p_{3}(s) & =\left[\frac{a \lambda(2 s+a+\lambda)}{(s+a)(s+\lambda)(s+\mu) I(s)}\right] \\
A_{v}(s) & =p_{0}(s)+p_{1}(s)=\left[\frac{(s+a+\lambda)}{(s+\lambda) I(s)}\right] \\
& =\left[\frac{\left(s^{3}+a_{2} s^{2}+a_{1} s+a_{0}\right)}{s\left(s^{3}+b_{2} s^{2}+b_{1} s+b_{0}\right)}\right]
\end{aligned}
$$

Where $a_{2}=(2 a+\lambda+\mu), a_{1}=\left(2 a \mu+a \lambda+a^{2}+\lambda \mu\right)$ and $a_{0}=\left(a \lambda \mu+a^{2} \mu\right)$

And $b_{2}=(2 \lambda+2 a+\mu), b_{1}=\left(2 a \mu+3 a \lambda+a^{2}+2 \lambda \mu+\lambda^{2}\right), b_{0}=\left(a \lambda^{2}+a^{2} \lambda+\lambda^{2} \mu+a \lambda \mu+a^{2} \mu\right)$

Taking inverse Laplace transforms of Eq. (32) we get

$$
\begin{aligned}
A_{v}(t) & =\left(\frac{-a_{0}}{s_{1} s_{2} s_{3}}\right)+\left(\frac{s_{1}{ }^{3}+a_{2} s_{1}{ }^{2}+a_{1} s_{1}+a_{0}}{s_{1}\left(s_{1}-s_{2}\right)\left(s_{1}-s_{3}\right)}\right) \exp \left(s_{1} t\right)+\left(\frac{s_{2}{ }^{3}+a_{2} s_{2}{ }^{2}+a_{1} s_{2}+a_{0}}{s_{2}\left(s_{2}-s_{1}\right)\left(s_{2}-s_{3}\right)}\right) \exp \left(s_{2} t\right) \\
& +\left(\frac{s_{3}{ }^{3}+a_{2} s_{3}{ }^{2}+a_{1} s_{3}+a_{0}}{s_{3}\left(s_{3}-s_{1}\right)\left(s_{3}-s_{2}\right)}\right) \exp \left(s_{3} t\right)
\end{aligned}
$$

Where $s_{1}, s_{2}$ and $s_{3}$ are roots of the Eq. $s^{3}+b_{2} s^{2}+b_{1} s+b_{0}=0$

\subsection{Busy Period of the repairman:}

Since $p_{3}(t)$ be the probability that the system is in failed state and the repairman remains busy in repairing the failed machine, therefore the busy period of the repairman $\mathrm{B}(\mathrm{t})$, during the interval $(0, t]$ is given by

$$
B(t)=\int_{0}^{t} p_{3}(t) d t
$$

Using Eq. (28) in Eq. (31), we get

$$
p_{3}(s)=\left[\frac{a \lambda(2 s+a+\lambda)}{s\left(s^{3}+b_{2} s^{2}+b_{1} s+b_{0}\right)}\right]
$$

Taking inverse Laplace transforms of Eq. (35) we get

$$
\begin{aligned}
p_{3}(t) & =\left(\frac{-a \lambda(a+\lambda)}{s_{1} s_{2} s_{3}}\right)+\left(\frac{a \lambda\left(2 s_{1}+a+\lambda\right)}{s_{1}\left(s_{1}-s_{2}\right)\left(s_{1}-s_{3}\right)}\right) \exp \left(s_{1} t\right)+\left(\frac{a \lambda\left(2 s_{2}+a+\lambda\right)}{s_{2}\left(s_{2}-s_{1}\right)\left(s_{2}-s_{3}\right)}\right) \exp \left(s_{2} t\right) \\
& +\left(\frac{a \lambda\left(2 s_{3}+a+\lambda\right)}{s_{3}\left(s_{3}-s_{1}\right)\left(s_{3}-s_{2}\right)}\right) \exp \left(s_{3} t\right)
\end{aligned}
$$

By using Eq. (36) in Eq, (34) and after solving, we get

$$
\begin{aligned}
B(t) & =\left(\frac{-a \lambda(a+\lambda) t}{s_{1} s_{2} s_{3}}\right)+\left(\frac{a \lambda\left(2 s_{1}+a+\lambda\right)}{s_{1}{ }^{2}\left(s_{1}-s_{2}\right)\left(s_{1}-s_{3}\right)}\right)\left(\exp \left(s_{1} t\right)-1\right)+\left(\frac{a \lambda\left(2 s_{2}+a+\lambda\right)}{s_{2}{ }^{2}\left(s_{2}-s_{1}\right)\left(s_{2}-s_{3}\right)}\right)\left(\exp \left(s_{2} t\right)-1\right) \\
& +\left(\frac{a \lambda\left(2 s_{3}+a+\lambda\right)}{s_{3}{ }^{2}\left(s_{3}-s_{1}\right)\left(s_{3}-s_{2}\right)}\right)\left(\exp \left(s_{3} t\right)-1\right)
\end{aligned}
$$




\subsection{Vacation time of the repairman:}

Since $p_{0}(t), p_{2}(t)$ be the probabilities that the system is in state 0 and 2 , where the repairman is on his vacation, therefore the vacation time of the repairman $\mathrm{V}(\mathrm{t})$, during the interval $(0, \mathrm{t}]$ is given by

$$
V(t)=\int_{0}^{t}\left\{p_{0}(t)+p_{2}(t)\right\} d t
$$

Now, by using Eq. (28) in Eqs. (27) and (30), we get

$$
p_{0}(s)+p_{2}(s)=\left[\frac{(s+a+\lambda)(s+\lambda)(s+\mu)}{s\left(s^{3}+b_{2} s^{2}+b_{1} s+b_{0}\right)}\right]
$$

Taking inverse Laplace transforms of Eq. (39) we get

$$
p_{0}(t)+p_{2}(t)=\left\{\begin{array}{l}
\left(\frac{-\mu \lambda(a+\lambda)}{s_{1} s_{2} s_{3}}\right)+\left(\frac{\left(s_{1}+a+\lambda\right)\left(s_{1}+\lambda\right)\left(s_{1}+\mu\right)}{s_{1}\left(s_{1}-s_{2}\right)\left(s_{1}-s_{3}\right)}\right) \exp \left(s_{1} t\right) \\
+\left(\frac{\left(s_{2}+a+\lambda\right)\left(s_{2}+\lambda\right)\left(s_{2}+\mu\right)}{s_{2}\left(s_{2}-s_{1}\right)\left(s_{2}-s_{3}\right)}\right) \exp \left(s_{2} t\right)+\left(\frac{\left(s_{3}+a+\lambda\right)\left(s_{3}+\lambda\right)\left(s_{3}+\mu\right)}{s_{3}\left(s_{3}-s_{1}\right)\left(s_{3}-s_{2}\right)}\right) \exp \left(s_{3} t\right)
\end{array}\right\}
$$

By using Eq. (40) in Eq, (38) and after solving, we get

$$
V(t)=\left\{\begin{array}{c}
\left(\frac{-\mu \lambda(a+\lambda) t}{s_{1} s_{2} s_{3}}\right)+\left(\frac{\left(s_{1}+a+\lambda\right)\left(s_{1}+\lambda\right)\left(s_{1}+\mu\right)}{s_{1}^{2}\left(s_{1}-s_{2}\right)\left(s_{1}-s_{3}\right)}\right)\left(\exp \left(s_{1} t\right)-1\right) \\
+\left(\frac{\left(s_{2}+a+\lambda\right)\left(s_{2}+\lambda\right)\left(s_{2}+\mu\right)}{s_{2}\left(s_{2}-s_{1}\right)\left(s_{2}-s_{3}\right)}\right)\left(\exp \left(s_{2} t\right)-1\right) \\
+\left(\frac{\left(s_{3}+a+\lambda\right)\left(s_{3}+\lambda\right)\left(s_{3}+\mu\right)}{s_{3}\left(s_{3}-s_{1}\right)\left(s_{3}-s_{2}\right)}\right)\left(\exp \left(s_{3} t\right)-1\right)
\end{array}\right\}
$$

\subsection{Idle time of the repairman:}

Since state 1 of the system represent that the machine being running well and the repairman remains idle, therefore the idle time of the repairman $I(t)$ during the interval $(0, t]$ is given by

$$
I(t)=\int_{0}^{t} p_{1}(t) d t
$$

Using Eq. (28) in Eq. (29), we get

$$
p_{1}(s)=\left[\frac{a(s+a)(s+\mu)}{s\left(s^{3}+b_{2} s^{2}+b_{1} s+b_{0}\right)}\right]
$$

Taking inverse Laplace transforms of Eq. (43) we get

$$
p_{1}(t)=\left\{\begin{array}{l}
\left(\frac{-a^{2} \mu}{s_{1} s_{2} s_{3}}\right)+\left(\frac{a\left(s_{1}+a\right)\left(s_{1}+\mu\right)}{s_{1}\left(s_{1}-s_{2}\right)\left(s_{1}-s_{3}\right)}\right) \exp \left(s_{1} t\right) \\
+\left(\frac{a\left(s_{2}+a\right)\left(s_{2}+\mu\right)}{s_{2}\left(s_{2}-s_{1}\right)\left(s_{2}-s_{3}\right)}\right) \exp \left(s_{2} t\right)+\left(\frac{a\left(s_{3}+a\right)\left(s_{3}+\mu\right)}{s_{3}\left(s_{3}-s_{1}\right)\left(s_{3}-s_{2}\right)}\right) \exp \left(s_{3} t\right)
\end{array}\right\}
$$

By using Eq. (44) in Eq, (42) and after solving, we get

$$
I(t)=\left\{\begin{array}{l}
\left(\frac{-a^{2} \mu t}{s_{1} s_{2} s_{3}}\right)+\left(\frac{a\left(s_{1}+a\right)\left(s_{1}+\mu\right)}{s_{1}^{2}\left(s_{1}-s_{2}\right)\left(s_{1}-s_{3}\right)}\right)\left(\exp \left(s_{1} t\right)-1\right) \\
+\left(\frac{a\left(s_{2}+a\right)\left(s_{2}+\mu\right)}{s_{2}^{2}\left(s_{2}-s_{1}\right)\left(s_{2}-s_{3}\right)}\right)\left(\exp \left(s_{2} t\right)-1\right)+\left(\frac{a\left(s_{3}+a\right)\left(s_{3}+\mu\right)}{s_{3}^{2}\left(s_{3}-s_{1}\right)\left(s_{3}-s_{2}\right)}\right)\left(\exp \left(s_{3} t\right)-1\right)
\end{array}\right\}
$$




\subsection{Profit analysis of the system:}

Let $K_{1}$ be the revenue per unit time and $K_{2}$ be the repair cost per unit time respectively, then the expected profit $E_{p}(t)$ during the interval $(0, t]$ is given by

$$
E_{p}(t)=K_{1} \int_{0}^{t} A_{v}(t) d t-K_{2} t
$$

By using Eq. (33) and after solving, we get

$$
E_{p}(t)=K_{1}\left[\begin{array}{l}
\left(\frac{-a_{0} t}{s_{1} s_{2} s_{3}}\right)+\left(\frac{s_{1}{ }^{3}+a_{2} s_{1}{ }^{2}+a_{1} s_{1}+a_{0}}{s_{1}{ }^{2}\left(s_{1}-s_{2}\right)\left(s_{1}-s_{3}\right)}\right)\left(\exp \left(s_{1} t\right)-1\right) \\
+\left(\frac{s_{2}{ }^{3}+a_{2} s_{2}{ }^{2}+a_{1} s_{2}+a_{0}}{s_{2}{ }^{2}\left(s_{2}-s_{1}\right)\left(s_{2}-s_{3}\right)}\right)\left(\exp \left(s_{2} t\right)-1\right) \\
+\left(\frac{s_{3}{ }^{3}+a_{2} s_{3}{ }^{2}+a_{1} s_{3}+a_{0}}{s_{3}{ }^{2}\left(s_{3}-s_{1}\right)\left(s_{3}-s_{2}\right)}\right)\left(\exp \left(s_{3} t\right)-1\right)
\end{array}\right]-K_{2} t
$$

Next, we have analyzed the performance of the system with respect to the various affecting parameters of the system which affects the system performance directly or indirectly. For it, the effect of the parameters namely failure rate $(\lambda)$ and arrival rate of the repairman $(a)$ on to the system reliability and the expected profit have been deducted. The results corresponding to these are summarized in Table 1 and Table 2 respectively for the reliability and the expected profit.

Table1. Effect of failure rate $(\lambda)$ on Reliability of the system $(R(t))$

\begin{tabular}{|l|l|l|l|}
\hline $\begin{array}{l}\text { Time } \\
(\mathrm{t})\end{array}$ & $\begin{array}{c}|c| \\
\lambda=0.02\end{array}$ & $\begin{array}{c}R(t) \\
\lambda=0.04\end{array}$ & $\begin{array}{c}R(t) \\
\lambda=0.06\end{array}$ \\
\hline 1 & 0.980199 & 0.960789 & 0.941765 \\
\hline 2 & 0.960789 & 0.923116 & 0.88692 \\
\hline 3 & 0.941765 & 0.88692 & 0.83527 \\
\hline 4 & 0.923116 & 0.852144 & 0.786628 \\
\hline 5 & 0.904837 & 0.818731 & 0.740818 \\
\hline 6 & 0.88692 & 0.786628 & 0.697676 \\
\hline 7 & 0.869358 & 0.755784 & 0.657047 \\
\hline 8 & 0.852144 & 0.726149 & 0.618783 \\
\hline 9 & 0.83527 & 0.697676 & 0.582748 \\
\hline 10 & 0.818731 & 0.67032 & 0.548812 \\
\hline
\end{tabular}


Table2. Effect of repair cost $\left(K_{2}\right)$ and arrival rate of repairman $(a)$ on expected profit (

$\left.E_{p}(t)\right)$
\begin{tabular}{|l|l|l|l|}
\hline $\begin{array}{l}\text { Time } \\
(\mathrm{t})\end{array}$ & $\begin{array}{l}K_{1}=1000, a=0.09, \\
\lambda=0.02, \mu=0.5\end{array}$ & $\begin{array}{l}K_{1}=1000, a=0.09, \\
\lambda=0.02, \mu=0.5\end{array}$ & $\begin{array}{l}K_{1}=1000, K_{2}=200, \\
\lambda=0.02, \mu=0.5\end{array}$ \\
& \multicolumn{1}{|c|}{$E_{p}(t)$} & $E_{p}(t)$ & \multicolumn{1}{c|}{$E_{p}(t)$} \\
& $\left(\right.$ for $\left.K_{2}=200\right)$ & $\left(\right.$ for $\left.K_{2}=150\right)$ & $($ for $a=0.1)$ \\
\hline 1 & 814.7071 & 864.7071 & 817.685 \\
\hline 2 & 1727.499 & 1827.499 & 1747.219 \\
\hline 3 & 2792.372 & 2942.372 & 2848.034 \\
\hline 4 & 4022.003 & 4222.003 & 4133.501 \\
\hline 5 & 5407.321 & 5657.321 & 5593.263 \\
\hline 6 & 6928.877 & 7228.877 & 7206.024 \\
\hline 7 & 8563.305 & 8913.305 & 8946.69 \\
\hline 8 & 10286.88 & 10686.88 & 10790.19 \\
\hline 9 & 12077.36 & 12527.36 & 12713.36 \\
\hline 10 & 13914.83 & 14414.83 & 14695.77 \\
\hline
\end{tabular}

However, in order to analyze the effect of the individual components on the system reliability and expected profit, an analysis has been conducted by varying a parameter at a time and simultaneously fixing the other parameters. The descriptions of these analyses have been summarized as follows.

If we increase the failure rate (i.e., $\lambda$ ) of the machine, then the corresponding reliability of the system is decreases. For instance, if we increase $\lambda$ from 0.02 to 0.04 and further from 0.04 to 0.06 then in both the cases, reliability of the system at a particular time, say 5 units, is decreased by $9.52 \%$. The complete variations of the effect of $\lambda$ on the system reliability with the passage of the time are summarized in Table-1.

On the other hand, we analyze the effect of the various parameters on to the expected profit $E_{p}(t)$ during the interval $(0, t]$ as given in Eq. (46). Firstly, we fix the different parameters as $\lambda=0.02, \mu=0.5$ and $K_{1}=500$. Now, the effect of the parameters namely repair cost $\left(K_{2}\right)$ and arrival rate of the repairman $(a)$ conducted an experiment in which the values of these parameters on $E_{p}(t)$ have been analyzed by fixing the values of the other component simultaneously with the passage of the time. The results corresponding to it have been summarized in table- 2 and details descriptions have been given as follows.

If we decrease the repair cost i.e., $K_{2}$ and fixing the other values, then their corresponding expected profit $E_{p}(t)$ is increases. For instance, if we decrease $K_{2}$ from 200 to 150 then expected profit at a particular time, say 5 units, is increased from 5407.321to 5657.321. Similarly, if we increase arrival rate of the repairman ( $a$ ) from 0.09 to 0.1 then their corresponding increase is 5593.263.

\section{Conclusion:}

In the present paper, we have proposed an approach for analyzing reliability and profit of a single-unit system with repairman having multiple vacations. In it, the unit works as new 
after its repair. Further, the effect of the various parameters on system reliability and expected profit have been analyzed and found that by varying $K_{2}$ and $a$, expected profit is increased. Based on it, the system analyst may focus on $K_{2}$ and $a$ parameters so as to increase the performance and productivity of the system. Hence, study reveals that a system will become economically beneficial; if we reduce the repair cost and increase the arrival rate of the repairman. In the future, we may apply it for two or more unit systems and for the complex repairable industrial systems.

\section{References:}

[1] B.H. Su and D.H. Shi, Reliability analysis of n-unit series systems with multiple vacations of a repairman, Math. Stat. Appl. Probab. Vol. 10, pp.78-82, 1995.

[2] B.T. Doshi, Queueing system with vacations- a survey, Queue. Syst. Vol.1, pp. 29-66, 1986.

[3] D. R. Cox, The analysis of non- Markovian stochastic process by the inclusion of supplementary variables, Proceedings of the Cambridge Philosophical Society, Vol. 51: pp.433-441, 1955.

[4] H. Sun and L. Ma, Modeling of a deteriorating system with single vacation and reliability analysis, Proceedings of the $33^{\text {rd }}$ Chinese Control Conference, pp. 3141-3146, 2014.

[5] H. Takagi, Queueing analysis: a foundation of performance evaluation, Vacation and priority systems, Part 1, North- Holland, Amsterdam, 1991.

[6] H. W. Lee, M/G/1 queue with exceptional first vacation, Comput. Oper. Res. Vol. 15 pp. 441-445, 1988.

[7] J.C. Ke, The optimal control of an M/G/1 queueing system with server startup and two vacation types, Appl. Math. Modell. Vol. 27, pp. 437-450, 2003.

[8] J. C. Ke, C. H. Wu and Z. G. Zhang, Recent developments in vacation models: a short survey, Int. J. Oper. Res. Vol. 7(4), pp. 3-8, 2010.

[9] J. Qiao and L. Guo, A repairable system with warning device and repairman vacation, Proceedings of the $27^{\text {th }}$ Chinese Control and Decision Conference (CCDC), pp. 39413947, 2015.

[10] L. Yuan and $\mathrm{J} . \mathrm{Xu}, \mathrm{A}$ deteriorating system with its repairman having multiple vacations, Appl. Math. Comput. Vol.217, pp. 4980-4989, 2011.

[11] M. S. Kadyan and Ramniwas, Cost benefit analysis of a single-unit system with warranty for repair, Appl. Math. Comput. Vol.223, pp. 346-353, 2013.

[12] M. Y. Zhang and Y. F. Wu, Reliability analysis of warm maintenance repairable system with continuous switch and multiple vacations for repairman, (in Chinese), J. Lanz. Univ. Techno. Vol. 41(5), pp. 152-157, 2015. 
[13] N. Tian and Z. G. Zhang, Vacation queueing models-theory and applications, Springer, New York, 2006.

[14] R. Niwas, M. S. Kadyan and J. Kumar, MTSF (mean time to system failure) and profit analysis of a single-unit system with inspection for feasibility of repair beyond warranty, Int. J. Syst. Assur. Eng. Manag. Vol. 7(1), pp.198-204, 2016.

[15] W. Q. Wu, Y. H. Tang and Y. Jiang, Study on a k-out-of-n: G repairable system with multiple vacations and one replaceable repair facility, (in Chinese), Syst. Eng. Theo. Pract. Vol. 33(10), pp. 2604-2614, 2013.

[16] Y. Levy, U. Yechiali; Utilization of the idle time in an M/G/1 queue, Manage. Sci. Vol.22, pp. 202-211, 1975. 